Fever induced by infection is regulated by components of the immune response, particularly the proinflammatory cytokines, interleukin-1B (IL-1B), IL-6, and tumor necrosis factor (TNF)-alpha, and the anti-inflammatory cytokine IL-10. Several recent studies have examined the levels of production of various components of the immune response in febrile children with and without seizures, with varying results. Despite some negative findings, most studies favor a cytokine role in febrile seizures. Serum levels of IL-6 and IL-10 are useful indicators of the severity of influenza-associated febrile seizures (Kawada J-I et al. $\mathbf{J}$ Infect Dis 2003;188:690-698; Millichap JG, Millichap JJ. J Infect Dis 2004;189:564-5; and idem Pediatr Neurol Sept 2006;35:165-172).

\title{
ELECTROCLINICAL FEATURES OF ABSENCE EPILEPSY
}

Clinical and EEG features of absence seizures in 47 children with newly diagnosed, untreated childhood absence epilepsy (CAE) were analyzed using video-EEG recordings, in a study at University of Otago, Wellington, New Zealand; British Columbia Children's Hospital, Vancouver, BC, Canada; and Royal Children's Hospital, Melbourne, Australia. CAE was defined as daily typical absence seizures presenting between the ages of 2 and 10 years, and without other seizure types. In 339 absence seizures analyzed, the average seizure duration was 9.4 seconds (range 1 to $44 \mathrm{sec}$ ). Clinical features included arrest of activity, loss of awareness, staring (upward in $33 \%$ patients, lateral in 28), and $3-\mathrm{Hz}$ eye blinking. Eye opening occurred in $59 \%$ of seizures, when eyes were initially closed. Automatisms, predominantly oral, were seen in $41 \%$ of seizures and $81 \%$ of patients. Hyperventilation induced seizures in $83 \%$ of patients. Ictal EEG showed regular $3-\mathrm{Hz}$ generalized spike and wave (GSW), with one or two spikes per wave, and postictal slowing. Interictal EEG showed GSW fragments (brief, $<2$ seconds, epileptiform discharges without clinical seizures), posterior bilateral delta activity (notched in $40 \%$ cases), and focal discharges. The recently proposed ILAE criteria for CAE were fulfilled by only 5 of the $47(11 \%)$ unselected patients in this study that used 1989 ILAE criteria. The authors conclude that the newly proposed criteria will be of limited value in the diagnosis of CAE and prediction of prognosis. (Sadleir LG, Farrell K, Smith S, Connolly MB, Scheffer LE. Electroclinical features of absence seizures in childhood absence epilepsy. Neurology August (1 of 2) 2006;67:413-418). (Reprints: Dr Lynette Grant Sadleir, Department of Paediatrics, Wellington School of Medicine and Health Sciences, University of Otago, PO Box 7343, Wellington, South New Zealand).

COMMENT. The clinical and EEG features of childhood absence epilepsy are heterogeneous, and the less restrictive 1989 ILAE diagnostic criteria used in the present study are of greater practical value than the criteria proposed in 2000 . The more recent criteria restrict the age to 4 to 10 years, seizure duration $>4 \mathrm{sec}$, no myoclonic features, no photic-induced seizures, $<3$ spikes per slow wave, and no disorganized discharges. CAE is defined as frequent daily absence seizures in otherwise normal school age children with EEG showing bilateral synchronous symmetric $3-\mathrm{Hz}$ spike-wave and normal background activity (ILAE Classification. Epilepsia 1989;30:389-399). The majority of children with CAE that fulfill these criteria have a good prognosis; $60 \%$ do not develop generalized tonic clonic seizures, and 95\% outgrow their absence seizures (Loiseau P, Panayiotopoulos C. 2002). 